\title{
DOES A BILLIARD ORBIT DETERMINE ITS (POLYGONAL) TABLE?
}

\author{
JOZEF BOBOK AND SERGE TROUBETZKOY
}

\begin{abstract}
We introduce a new equivalence relation on the set of all polygonal billiards. We say that two billiards (or polygons) are order equivalent if each of the billiards has an orbit whose footpoints are dense in the boundary and the two sequences of footpoints of these orbits have the same combinatorial order. We study this equivalence relation with additional regularity conditions on the orbit.
\end{abstract}

\section{INTRODUCTION}

In mathematics one often wants to know if one can reconstruct a object (often a geometric object) from certain discrete data. A famous example of this is the celebrated problem posed by Mark Kac, "Can one hear the shape of a drum", i.e., whether one can reconstruct a drum head from knowing the frequencies at which it vibrates [K]. This problem was resolved negatively by Milnor in dimension 16 [M] and then by Gordon, Webb and Wolpert in dimension 2 [GWW]. Another well known example is a question posed by Burns and Katok whether a negatively curved surface is determined by its marked length spectrum [BK]. The "marked length spectrum" of a surface $S$ is the function that associates to each conjugacy class in $\pi_{1}(S)$ the length of the geodesic in the associated free homotopy class. This question was resolved positively by Otal $[\mathrm{O}]$.

In this article we ask if a polygonal billiard table is determined by the combinatorial data of the footpoints of a billiard orbit. For this purpose we introduce a new equivalence relation on the set of all polygonal billiards. Namely we say that two polygonal billiards (polygons) are order equivalent if each of the billiards has an orbit whose footpoints are dense in the boundary and the two sequences of footpoints of these orbits have the same combinatorial order. We study this equivalence relation with additional regularity conditions on the orbit. Our main results are the following, under a weak regularity condition on the orbits, an irrational polygon can not be order equivalent to a rational polygon and two order equivalent rational polygons must have the same 
number of sides with corresponding corners having the same angle. In the case of triangles, only similar triangles can be order equivalent. In general, in the rational case, one can not say more since any two rectangles are order equivalent. If we furthermore assume that the greatest common denominator of the rational angles is at least 3, then under a slightly stronger regularity condition we show that the two order equivalent rational polygons must be similar. In the case that the greatest common denominator is 2 , the two order equivalent rational polygons must be affinely similar.

In Section 2 we start by recalling basic needed facts about polygonal billiards while in Section 3 we prove various preparatory lemmas. We prove our main results in Sections 4 (rational versus irrational) and 5 (rational versus rational). Finally in Section 6 we summerize our results and ask some open questions.

\section{Polygonal Billiard}

A polygonal billiard table is a planar simply connected compact polygon $P$. The billiard flow $\left\{T_{t}\right\}_{t \in \mathbb{R}}$ in $P$ is generated by the free motion of a mass-point subject to the elastic reflection in the boundary. This means that the point moves along a straight line in $P$ with a constant speed until it hits the boundary. At a smooth boundary point the billiard ball reflects according to the well known law of geometrical optics: the angle of incidence equals to the angle of reflection. If the billiard ball hits a corner, (a non-smooth boundary point), its further motion is not defined. Additionally to a corner, the billiard trajectory is not defined for a direction tangent to a side.

By $D$ we denote the group generated by the reflections in the lines through the origin, parallel to the sides of the polygon $P$. The group $D$ is either

- finite, when all the angles of $P$ are of the form $\pi m_{i} / n_{i}$ with distinct co-prime integers $m_{i}, n_{i}$, in this case $D=D_{N}$ the dihedral group generated by the reflections in lines through the origin that meet at angles $\pi / N$, where $N$ is the least common multiple of $n_{i}$ 's,

or

- countably infinite, when at least one angle between sides of $P$ is an irrational multiple of $\pi$.

In the two cases we will refer to the polygon as rational respectively irrational.

Consider the phase space $P \times S^{1}$ of the billiard flow $T_{t}$, and for $\theta \in S^{1}$, let $R_{\theta}$ be its subset of points whose second coordinate belongs 
to the orbit of $\theta$ under $D$. Since a trajectory changes its direction by an element of $D$ under each reflection, $R_{\theta}$ is an invariant set of the billiard flow $T_{t}$ in $P$.

The billiard map $T: V_{P}=\cup e \times \theta \subset \delta P \times S^{1} \rightarrow V_{P}$ associated with the flow $T_{t}$ is the first return map to the boundary $\delta P$ of $P$. Here the union $\cup e \times \theta$ is taken over all sides of $P$ and for each side $e$ over the inner pointing directions $\theta$ from $S^{1}$. We will denote points of $V_{P}$ by $u=(x, \theta)$.

The set $P \times \theta$, resp. $\delta P \times \theta$ will be called a floor of the phase space of the flow $T_{t}$, resp. of the billiard map $T$.

Let $\delta P$ be oriented counterclockwise. For $x, x^{\prime} \in \delta P$, by $\left[x, x^{\prime}\right]$ $\left(\left(x, x^{\prime}\right)\right)$ we denote a closed (open) arc with outgoing endpoint $x$ and incoming endpoint $x^{\prime}$.

If $P, Q$ are simply connected polygons with counterclockwise oriented boundaries, two sequences $\left\{x_{n}\right\}_{n \geq 0} \subset \delta P$ and $\left\{y_{n}\right\}_{n \geq 0} \subset \delta Q$ have the same combinatorial order if for each non-negative integers $k, l, m$

$$
x_{k} \in\left[x_{l}, x_{m}\right] \Longleftrightarrow y_{k} \in\left[y_{l}, y_{m}\right] .
$$

The next definition introduces a new relation on the set of all simply connected polygons. The reader can verify that it is reflexive, symmetric and transitive, i.e., it is an equivalence relation. As usual, $\pi_{1}$ denotes the natural projection to the first coordinate (the foot point).

Definition 2.1. We say that two polygons (or polygonal billiards) $P, Q$ are order equivalent if there are points $u_{0} \in V_{P}, v_{0} \in V_{Q}$ such that

(i) ${\overline{\left\{\pi_{1}\left(T^{n}\left(u_{0}\right)\right)\right\}_{n>0}}}_{n>}=\delta,{\overline{\left\{\pi_{1}\left(S^{n}\left(v_{0}\right)\right)\right\}_{n>0}}}_{n}=\delta Q$,

(ii) the sequences $\left\{\pi_{1}\left(T^{n}\left(u_{0}\right)\right)\right\}_{n \geq 0},\left\{\pi_{1}\left(S^{n}\left(v_{0}\right)\right)\right\}_{n \geq 0}$ have the same combinatorial order.

In such a case we will write $\left(P ; u_{0}\right) \approx\left(Q ; v_{0}\right)$, the points $u_{0}, v_{0}$ will be sometimes called the leaders.

Let $t=\left\{x_{n}\right\}_{n \geq 0}$ be a sequence which is dense in $\delta P$. The $t$-address $a_{t}(x)$ of a point $x \in \delta P$ is the set of all increasing sequences $\{n(k)\}_{k}$ of non-negative integers satisfying $\lim _{k} x_{n(k)}=x$. It is clear that any $x \in \delta P$ has a nonempty $t$-address and $t$-addresses of two distinct points from $\delta P$ are disjoint.

For order equivalent polygons $P, Q$ with leaders $u_{0}, v_{0}$, we will consider addresses with respect to the sequences given by Definition 2.1(ii):

$$
t=\left\{\pi_{1}\left(T^{n}\left(u_{0}\right)\right)\right\}_{n \geq 0}, s=\left\{\pi_{1}\left(S^{n}\left(v_{0}\right)\right)\right\}_{n \geq 0} .
$$

It is an easy exercise to prove that the map $\phi: \delta P \rightarrow \delta Q$ defined by

$$
\phi(x)=y \text { if } a_{t}(x)=a_{s}(y)
$$


is a homeomorphism.

Concerning the order-equivalence of polygonal billiards, the reader can ask possible properties of corresponding equivalence classes. In particular, can rational and irrational polygons simultaneously lie in the same class? Can order equivalent polygons have different (number of) angles, or at least different lengths of sides?

It follows immediately from the well known results that order equivalent polygons need not be similar (see for example [MT]).

Example 2.2. Any two rectangles are order equivalent.

We finish this section recalling several well known and useful (for our purpose) results about polygonal billiards (see for example [MT]). Recall that a flat strip $\mathcal{T}$ is an invariant subset of the phase space of the billiard flow/map such that

1) $\mathcal{T}$ is contained in a finite number of floors,

2) the billiard flow/map dynamics on $\mathcal{T}$ is minimal in the sense that any orbit which does not hit a corder is dense in $\mathcal{T}$,

3) the boundary of $\mathcal{T}$ is non-empty and consists of a finite union of generalized diagonals (a generalized diagonal is a billiard trajectory that goes from a corner to a corner).

By $\pi_{2}$ we denote the second natural projection (to the direction). A direction, resp. a point $u$ from the phase space is exceptional if it is the direction of a generalized diagonal, resp. $\pi_{2}(u)$ is such a direction. Obviously there are countably many generalized diagonals hence also exceptional directions. A direction, resp. a point $u$ from the phase space, which is not exceptional will be called non-exceptional.

The set of the corners of $P$ is denoted by $C_{P}$.

Proposition 2.3. [MT] Let $P$ be rational and $u_{0} \in V_{P}$. Then exactly one of the following three possibilities has to be satisfied.

(i) $u_{0}$ is periodic.

(ii) $\overline{\operatorname{orb}}\left(u_{0}\right)$ is a flat strip.

(iii) $\omega\left(u_{0}\right)=R_{\pi_{2}\left(u_{0}\right)}$; then

$$
\#\left(\left\{\pi_{2}\left(T^{n}\left(u_{0}\right)\right): n \geq 0\right\}\right)=2 N,
$$

and for every $x \in \delta P \backslash C_{P}$,

$$
\#\left\{u \in \omega\left(u_{0}\right): \pi_{1}(u)=x\right\}=N,
$$

where $N$ is the least common multiple of the denominators of angles of $P$. Moreover, in this case

$$
\pi_{2}\left(\left\{u \in \omega\left(u_{0}\right): \pi_{1}(u)=x\right\}\right)=\pi_{2}\left(\left\{u \in \omega\left(u_{0}\right): \pi_{1}(u)=x^{\prime}\right\}\right)
$$


whenever $x^{\prime} \notin C_{P}$ belongs to the same side as $x$. The possibility (iii) is true whenever $u_{0}$ is non-exceptional.

The billiard map $T$ has a natural invariant measure on its phase space given by the formula $\mu=\sin \theta \mathrm{d} x \mathrm{~d} \theta$, where $\theta \in(0, \pi)$ is measured with respect to the direction of an oriented side $e$. In a rational polygon we say that a point $u$ is generic if it is non-exceptional, has bi-infinite orbit and the billiard map restricted to the invariant surface $R_{\pi_{2}(u)}$ has a single invariant measure (this measure is then automatically the measure $\mu$ ).

Finally we will use the following remarkable result from [V].

Theorem 2.4. MT] If $P$ is a regular $n$-gon $(n \neq 3)$ then any nonexceptional $u$ with infinite orbit is generic. Any exceptional $u$, the trajectory of which does not hit a corner, is periodic.

\section{Preparatory Lemmas}

To define some important objects in dynamical systems, for example an $\omega$-limit set - the corresponding phase space has to be equipped with a topology providing suitable convergence. In the context of polygonal billiards it often means the simultaneous convergence of foot points and directions (shortly pointwise convergence). Since it simplifies several our proofs, throughout the whole paper we will use the following modified definition of convergence.

Definition 3.1. Let $\left\{T^{n}\left(u_{0}\right)\right\}_{n \geq 0}$ be an infinite trajectory. The sequence $\left\{T^{n(k)}\left(u_{0}\right)\right\}_{k \geq 0}$ converges if and only if

(i) $\pi_{1}\left(T^{n(k)}\left(u_{0}\right)\right) \rightarrow_{k} x \in \delta P$,

(ii) $\pi_{1}\left(T^{n(k)+1}\left(u_{0}\right)\right) \rightarrow_{k} x^{\prime} \in \delta P$,

(iii) the points $x$ and $x^{\prime}$ do not belong to the same side of $P$.

In particular, the above definition omits the case when a sequence $\left\{T^{n(k)}\left(u_{0}\right)\right\}_{k \geq 0}$ satisfies (i),(ii) but $x=x^{\prime}$. Not to rewrite known results in slightly modified versions we will use the following

Fact 3.2. Despite the fact that this notion is stronger than the usual convergence we will use classical results, such as Proposition 2.3(ii),(iii). All the results we use remain true under our notion of convergence.

Lemma 3.3. Let $\left(P ; u_{0}\right) \approx\left(Q ; v_{0}\right)$, assume that to any point $x^{\prime} \in$ $C_{P} \cup \phi^{-1}\left(C_{Q}\right)$ there exists a sequence $\{n(k)\}_{k \geq 1}$ such that the items (i),(ii) of Definition 3.1 are satisfied, $x \neq x^{\prime}$ and $x^{\prime}$ is a both-side limit point of $\left\{\pi_{1}\left(T^{n(k)+1}\left(u_{0}\right)\right)\right\}_{k \geq 0}$. Then $\phi\left(C_{P}\right)=C_{Q}$. 
Proof. Choose $x^{\prime} \in C_{P} \backslash \phi^{-1}\left(C_{Q}\right)$. Let $\left\{T^{n(k)}\left(u_{0}\right)\right\}_{k \geq 0}$ be a sequence satisfying the assumptions of the lemma. Consider its 'counterpart' in $Q,\left\{S^{n(k)}\left(v_{0}\right)\right\}_{k \geq 0}$. Let

$$
y=\phi(x)=\lim _{k} \pi_{1}\left(S^{n(k)}\left(v_{0}\right)\right), y^{\prime}=\phi\left(x^{\prime}\right)=\lim _{k} \pi_{1}\left(S^{n(k)+1}\left(v_{0}\right)\right) .
$$

Then $\lim _{k} \pi_{1}\left(T^{n(k)+2}\left(u_{0}\right)\right)$ does not exist since $x^{\prime}$ is a corner and a bothsided limit. However $\lim _{k} \pi_{1}\left(S^{n(k)+2}\left(v_{0}\right)\right)$ does exist, this is impossible for order equivalent polygons $\left(P ; u_{0}\right) \approx\left(Q ; v_{0}\right)$.

The case when $x^{\prime} \in \phi^{-1}\left(C_{Q}\right) \backslash C_{P}$ can be excluded analogously. Thus, $C_{P}=\phi^{-1}\left(C_{Q}\right)$, i.e., $\phi\left(C_{P}\right)=C_{Q}$.

As usual, an $\omega$-limit set of a point $u$ is denoted by $\omega(u)$. Let us define the map $\Phi:\left\{T^{n}\left(u_{0}\right)\right\}_{n \geq 0} \rightarrow\left\{S^{n}\left(v_{0}\right)\right\}_{n \geq 0}$ by

$$
\Phi\left(T^{n}\left(u_{0}\right)\right)=S^{n}\left(v_{0}\right) .
$$

Lemma 3.4. Let $\left(P ; u_{0}\right) \approx\left(Q ; v_{0}\right)$ and $\phi\left(C_{P}\right)=C_{Q}$. The following hold

(i) $T^{n(k)}\left(u_{0}\right) \rightarrow_{k} u \in V_{P}$ if and only if $S^{n(k)}\left(v_{0}\right) \rightarrow_{k} v \in V_{Q}$.

(ii) The map $\Phi$ from (2) can be extended homeomorphically to the map $\Phi: \omega\left(u_{0}\right) \rightarrow \omega\left(v_{0}\right)$ satisfying (for all $n \in \mathbb{Z}$ for which the image is defined)

$$
\Phi\left(T^{n}(u)\right)=S^{n}(\Phi(u)), u \in \omega\left(u_{0}\right) ;
$$

in particular, for every $x \in \delta P$ we have

$$
\Phi\left(\left\{u \in \omega\left(u_{0}\right): \pi_{1}(u)=x\right\}\right)=\left\{v \in \omega\left(v_{0}\right): \pi_{1}(v)=\phi(x)\right\} .
$$

(iii) For every $u, u^{\prime} \in \omega\left(u_{0}\right)$ with $\pi_{1}(u)=\pi_{1}\left(u^{\prime}\right) \notin C_{P}$,

$$
\pi_{2}(u)<\pi_{2}\left(u^{\prime}\right) \Longleftrightarrow \pi_{2}(\Phi(u))<\pi_{2}\left(\Phi\left(u^{\prime}\right)\right),
$$

(iv) $u_{0}$ is recurrent if and only if $v_{0}$ is recurrent.

Proof. (i) Let $T^{n(k)}\left(u_{0}\right) \rightarrow_{k} u$ and $x, x^{\prime}$ be points from Definition 3.1. Then both the limits $y=\phi(x)=\lim _{k} \pi_{1}\left(S^{n(k)}\left(v_{0}\right)\right)$ and $y^{\prime}=\phi\left(x^{\prime}\right)=$ $\lim _{k} \pi_{1}\left(S^{n(k)+1}\left(v_{0}\right)\right)$ exist and, since $\phi\left(C_{P}\right)=C_{Q}$, the points $y, y^{\prime}$ cannot lie on the same side of $Q$. Thus $S^{n(k)}\left(v_{0}\right) \rightarrow_{k} v$ where $v$ is the unit vector with foot point $y$ and pointing towards $y^{\prime}$. The opposite implication is symmetric.

(ii) Denote $u_{n}=T^{n}\left(u_{0}\right)$. For $u \in \omega\left(u_{0}\right)$ for which $u=\lim _{k} u_{n(k)}$ put

$$
\Phi(u)=\lim _{k} \Phi\left(u_{n(k)}\right) ;
$$

using (2) and part (i) of the lemma one can easily show that $\Phi$ is well defined and a homeomorphism. Thus (3) follows. 
(iii) Since the homeomorphism $\phi$ preserves the corners, we have $\pi_{1}(\Phi(u))=\pi_{1}\left(\Phi\left(u^{\prime}\right)\right) \notin C_{Q}$. From (ii) we obtain that $\pi_{2}(u) \neq \pi_{2}\left(u^{\prime}\right)$ if and only if $\pi_{2}(\Phi(u)) \neq \pi_{2}\left(\Phi\left(u^{\prime}\right)\right)$. If (4) does not hold then there are positive integers $n, n^{\prime}$ such that $u_{n}$, resp. $u_{n^{\prime}}$ is close to $u$, resp. $u^{\prime}$ (with the foot points on the same side, in particular) and either

$$
\pi_{2}\left(u_{n}\right)<\pi_{2}\left(u_{n^{\prime}}\right) \& \pi_{2}\left(\Phi\left(u_{n}\right)\right)>\pi_{2}\left(\Phi\left(u_{n^{\prime}}\right)\right),
$$

or an analogous possibility with changed signs of inequality is true. Without loss of generality consider the first possibility. Then for the corresponding arcs

$$
\left[\pi_{1}\left(u_{n^{\prime}+1}\right), \pi_{1}(u)\right) \cap\left(\pi_{1}(u), \pi_{1}\left(u_{n+1}\right)\right]=\emptyset
$$

and

$$
\begin{gathered}
{\left[\pi_{1}\left(\Phi\left(u_{n^{\prime}+1}\right)\right), \pi_{1}(\Phi(u))\right) \cap\left(\pi_{1}(\Phi(u)), \pi_{1}\left(\Phi\left(u_{n+1}\right)\right)\right]=} \\
=\left[\pi_{1}\left(\Phi\left(u_{n^{\prime}+1}\right)\right), \pi_{1}\left(\Phi\left(u_{n+1}\right)\right)\right] \neq \emptyset,
\end{gathered}
$$

what is impossible for order equivalent polygons $\left(P ; u_{0}\right) \approx\left(Q ; v_{0}\right)$.

Part (iv) easily follows from (i).

Let $g$ be a function defined on a neighborhood of $y$. The derived numbers $D^{+} g(y), D_{+} g(y)$ of $g$ at $y$ are given by

$$
D^{+} g(y)=\limsup _{h \rightarrow 0_{+}} \frac{g(y+h)-g(y)}{h}, D_{+} g(y)=\liminf _{h \rightarrow 0_{+}} \frac{g(y+h)-g(y)}{h}
$$

and the analogous limits from the left are denoted by $D^{-} g(y), D_{-} g(y)$.

Let $(z, y)$ be the coordinates of $\mathbb{R}^{2}$ and let $p_{a, b} \subset \mathbb{R}^{2}$ be the line with equation $y=a+z \tan b$. For short we denote $p_{y_{0}, g\left(y_{0}\right)}$ by $p_{g\left(y_{0}\right)}$.

Lemma 3.5. Let $g:(c, d) \rightarrow\left(-\frac{\pi}{2}, \frac{\pi}{2}\right)$ be a continuous function, fix $C \subset$ $(c, d)$ countable. Assume that for some $y_{0}$ one of the four possibilities

$$
D^{+} g\left(y_{0}\right)>0, D_{+} g\left(y_{0}\right)<0, D^{-} g\left(y_{0}\right)>0, D_{-} g\left(y_{0}\right)<0
$$

is fulfilled. Then there exists a sequence $\left\{y_{n}\right\}_{n \geq 1} \subset(c, d) \backslash C$ such that $\lim _{n} y_{n}=y_{0}$ and the set of crossing points $\left\{p_{g\left(y_{0}\right)} \cap p_{g\left(y_{n}\right)}: n \geq 1\right\}$ is bounded in the $\mathbb{R}^{2}$.

Proof. We will prove the conclusion when $\epsilon=D^{+} g\left(y_{0}\right)>0$. The three remaining possibilities can be shown analogously.

By our assumption there exists a decreasing sequence $\left\{y_{n}\right\}_{n \geq 1} \subset$ $(c, d) \backslash C$ such that $\lim _{n} y_{n}=y_{0}$ and for each $n$

$$
g\left(y_{n}\right)>g_{n}=g\left(y_{0}\right)+\frac{\epsilon}{2}\left(y_{n}-y_{0}\right)>g\left(y_{0}\right)
$$


It means that the crossing point $p_{g\left(y_{0}\right)} \cap p_{g\left(y_{n}\right)}$ is closer to the point $\left(0, y_{0}\right)$ than the crossing point $A_{n}=p_{g\left(y_{0}\right)} \cap p_{y_{n}, g_{n}}$. The first coordinate of $A_{n}=\left(\tilde{z}_{n}, \tilde{y}_{n}\right)$ satisfies

$$
y_{0}+\tilde{z}_{n} \tan g\left(y_{0}\right)=y_{n}+\tilde{z}_{n} \tan g_{n}
$$

hence

Since

$$
\tilde{z}_{n}=\frac{y_{0}-y_{n}}{\tan \left[g\left(y_{0}\right)+\frac{\epsilon}{2}\left(y_{n}-y_{0}\right)\right]-\tan g\left(y_{0}\right)} .
$$

$$
\lim _{n} \tilde{z}_{n}=\frac{-2}{\epsilon} \cos ^{2} g\left(y_{0}\right)
$$

both the sets $\left\{A_{n}: n \geq 1\right\},\left\{p_{g\left(y_{0}\right)} \cap p_{g\left(y_{n}\right)}: n \geq 1\right\}$ are bounded in the $\mathbb{R}^{2}$.

Remark 3.6. Note that if $g^{\prime}\left(y_{0}\right) \neq 0$ at some point $y_{0}$ then at least one of the four possibilities in (5) has to be fulfilled.

To apply this lemma to billiards, we remind the reader that notion of an unfolded billiard trajectory. Namely, instead of reflecting it in a side of $P$ one may reflect $P$ in this side and unfold the trajectory to a straight line.

Lemma 3.7. Let $\left(P ; u_{0}\right) \approx\left(Q ; v_{0}\right)$ with $P$ rational. Then the set of directions

$$
\left\{\pi_{2}\left(S^{n}\left(v_{0}\right)\right): n \geq 0\right\}
$$

along the trajectory of $v_{0}$ is finite.

Remark 3.8. In Lemma 3.7 we do not assume $u_{0}$ is non-exceptional.

Proof. We know that $\phi(\delta P)=\delta Q$, where $\phi$ is a homeomorphism defined in (1). Under our assumption we cannot exclude the possibility $\phi\left(C_{P}\right) \neq C_{Q}$. So, it will be convenient to consider extended sets of corners, $C_{P}^{*}, C_{Q}^{*}$, defined as

$$
C_{P}^{*}=\left\{x \in \delta P: x \in C_{P} \text { or } \phi(x) \in C_{Q}\right\}, C_{Q}^{*}=\phi\left(C_{P}^{*}\right),
$$

and the sides of $P$, resp. $Q$ will be taken with respect to $C_{P}^{*}$, resp. $C_{Q}^{*}$.

By Definition 2.1(i) the first projection of the forward trajectory of $u_{0}$ is dense in $\delta P$, so in particular, $u_{0}$ is not periodic. In such a case the trajectory of $u_{0}$ is minimal either in a flat strip $\mathcal{T}$ or an invariant surface $R_{\pi_{2}\left(u_{0}\right)}$ - see Proposition 2.3 . Since the conclusion can be verified for both possibilities in a similar way, we will only treat the flat strip $\mathcal{T}$ case.

For an interval $(a, b) \subset \delta P$ and an $\alpha \in S^{1}$ denote

$$
I:=\left\{n \in \mathbb{N}_{0}: \pi_{1}\left(T^{n}\left(u_{0}\right)\right) \in(a, b) \& \pi_{2}\left(T^{n}\left(u_{0}\right)\right)=\alpha\right\} .
$$


By Proposition 2.3 there are finitely many directions along the trajectory of $u_{0}$ and, by our assumption, the foot points of it are dense in $\delta P$; passing to a subinterval and choosing a suitable $\alpha$ we can assume that $(a, b)$, resp. $\phi((a, b))=(c, d)$ is a subinterval of a "side" $e$ of $P$, resp. a "side" $f$ of $Q$ and the sequence $\left\{\pi_{1}\left(T^{n}\left(u_{0}\right)\right)\right\}_{n \in I}$ is dense in $(a, b)$. Obviously,

$$
\tau=(a, b) \times\{\alpha\} \subset \omega\left(u_{0}\right), \sigma=\Phi(\tau) \subset \omega\left(v_{0}\right) ;
$$

there is a countable subset $\tau_{0}$ of $\tau$ such that each point from $\tau \backslash \tau_{0}$ has a bi-infinite trajectory (either the forward or backward trajectory starting from any point of $\tau_{0}$ finishes in a corner from $\left.C_{P}^{*}\right)$.

Clearly, the sequence $\left\{\pi_{1}\left(S^{n}\left(v_{0}\right)\right)\right\}_{n \in I}$ is dense in $(c, d)=\phi((a, b))$. Define a continuous function $g:(c, d) \rightarrow S^{1}$ by

$$
g(y)=\pi_{2}\left(\Phi\left(\phi^{-1}(y) \times\{\alpha\}\right)\right) .
$$

In what follows we will show that since the polygons $P, Q$ are order equivalent, the continuous function $g$ has to be constant. It is sufficient to show that $g^{\prime}\left(y_{0}\right)=0$ whenever $y_{0} \in(c, d) \backslash C$, where $C=\pi_{1}\left(\Phi\left(\tau_{0}\right)\right)$ is countable. Choose the origin of $S^{1}$ to be the perpendicular direction of the side of $Q$ containing $(c, d)$ and fix $y_{0} \in(c, d) \backslash C$; then for a sufficiently small neighborhood $U\left(y_{0}\right)$ of $y_{0}, g\left(U\left(y_{0}\right)\right) \subset\left(-\frac{\pi}{2}, \frac{\pi}{2}\right)$.

For $y^{\prime} \in U\left(y_{0}\right) \backslash C$ consider the unfolded (bi-infinite) billiard trajectory of $\left(y^{\prime}, g\left(y^{\prime}\right)\right)$ under the billiard flow $\left\{S_{t}\right\}_{t \in \mathbb{R}}$ in $Q$. Via unfolding, this trajectory corresponds to the line $p_{g\left(y^{\prime}\right)}$ with the equation $y=y^{\prime}+z \tan g\left(y^{\prime}\right)$.

Claim 3.9. There is no sequence $\left\{y_{n}\right\}_{n \geq 1} \subset(c, d) \backslash C$ such that $\lim _{n} y_{n}=$ $y_{0}$ and the set of crossing points $\left\{p_{g\left(y_{0}\right)} \cap p_{g\left(y_{n}\right)}: n \geq 1\right\}$ is bounded.

Proof. Denote by $S^{m}(y)$ the copy of $S^{m}((y, g(y)))$ on $p_{g(y)}$. To the contrary, let $\left\{y_{n}\right\}_{n \geq 1} \subset(c, d) \backslash C$ satisfy $\lim _{n} y_{n}=y_{0}$ and the set of crossing points $\left\{p_{g\left(y_{0}\right)} \cap p_{g\left(y_{n}\right)}: n \geq 1\right\}$ is bounded. Without loss of generality we can assume that the crossing points lie in the positive part of $p_{g\left(y_{0}\right)}$ and that there exists a value $m$ for which all crossing points from $\left\{p_{g\left(y_{0}\right)} \cap p_{g\left(y_{n}\right)}: n \geq 1\right\}$ lie on the link $\left(S^{m-1}\left(y_{0}\right), S^{m}\left(y_{0}\right)\right) \subset p_{g\left(y_{0}\right)}$. Choose a point $y_{n}$ such that each two points

$$
S^{i}\left(y_{0}\right), S^{i}\left(y_{n}\right), i=0, \ldots, m
$$

lie on the same side of $Q$ and the crossing point $p_{g\left(y_{0}\right)} \cap p_{g\left(y_{n}\right)}$ lies on the link $\left(S^{m-1}\left(y_{n}\right), S^{m}\left(y_{n}\right)\right) \subset p_{g\left(y_{n}\right)}$. Such a $y_{n}$ does exist since the function $g$ is continuous and $p_{g\left(y_{0}\right)}$ does not contain any copy of a corner from $C_{Q}^{*}$. Thus,

$$
\left(S^{m-1}\left(y_{0}\right), S^{m}\left(y_{0}\right)\right) \cap\left(S^{m-1}\left(y_{n}\right), S^{m}\left(y_{n}\right)\right) \neq \emptyset
$$


At the same time, for the points $x_{0}=\phi^{-1}\left(y_{0}\right), x_{n}=\phi^{-1}\left(y_{n}\right), \lim _{n} x_{n}=$ $x_{0}$, the iterates $T^{i}\left(\left(x_{0}, \alpha\right)\right), T^{i}\left(\left(x_{n}, \alpha\right)\right)$ lie on the same side of $P$, $i=0, \ldots, m$, and the unfolded both-side billiard trajectories of $\left(x_{0}, \alpha\right)$, $\left(x_{n}, \alpha\right)$ under the billiard flow $\left\{T_{t}\right\}_{t \in \mathbb{R}}$ in $P$ are parallel lines. In particular for the links,

$$
\left(T^{m-1}\left(x_{0}\right), T^{m}\left(x_{0}\right)\right) \cap\left(T^{m-1}\left(x_{n}\right), T^{m}\left(x_{n}\right)\right)=\emptyset,
$$

what contradicts (8) for order equivalent polygons $\left(P ; u_{0}\right) \approx\left(Q ; v_{0}\right)$ and the claim is proved.

Applying Lemma 3.5 and Claim 3.9 we obtain that the function $g$ defined in (7) satisfies $g^{\prime}\left(y_{0}\right)=0$ for every $y_{0} \in(c, d) \backslash C$ hence as a continuous function has to be constant. It means that

$$
S^{n}\left(v_{0}\right) \in \sigma \text { and } \pi_{2}\left(S^{n}\left(v_{0}\right)\right)=g\left(y_{0}\right) \text { whenever } n \in I
$$

and $I$ is given by (6) . We assumed that the trajectory of $u_{0}$ is minimal in a flat strip $\mathcal{T}$. It is known that in such a case the gaps in $I$ are bounded. This fact together with (9) imply that the set of directions $\left\{\pi_{2}\left(S^{n}\left(v_{0}\right)\right): n \geq 0\right\}$ along the trajectory of $v_{0}$ is finite. This proves the lemma.

\section{RAtional VERsus IrRATiOnAL}

As in Section 2, by $D$ we denote the group generated by the reflections in the lines through the origin, parallel to the sides of the polygon $P$. The group $D$ is (countable) infinite if and only if the polygon $P$ is irrational [MT]. In our case when $P$ is simply connected it is equivalent to the condition that some angle of $P$ is not $\pi$-rational.

Theorem 4.1. Let $P$ be irrational, $u$ a point from the phase space.

(i) If the orbit of $u$ does not hit a corner and $\theta=\pi_{2}(u)$ is nonexceptional then $\left\{\pi_{2}\left(T^{n}(u)\right): n \geq 0\right\}$ is infinite.

(ii) If $u$ is not periodic, but visits only a finite number of floors then ( $u$ is recurrent and) $\overline{\text { orb }}(u)$ is a flat strip.

Proof. (i) Fix $P$ and $\theta$ as in the statement of the lemma. Enumerate the infinite orbit $D \theta$ by $\left\{\theta_{1}, \theta_{2}, \ldots\right\}$. Consider $I:=\cup e_{j} \times \theta_{i}$. This union is taken over all sides and for each side over the inner pointing (at some interior point of the side) $\theta_{i}$ from $S^{1}$. Equip each $e_{j} \times \theta_{i}$ by a measure $\sin \theta_{i} d x$ where $d x$ is the arc length along $e_{j}$ and $\theta_{i} \in(0, \pi)$ is measured with respect to the oriented side $e_{j}$. We will refer to this measure as length. The billiard map $T$ leaves $I$ invariant and acts as an infinite interval exchange map. The boundary point of each interval of continuity corresponds to a (first) preimage of a corner of $P$. 
Suppose that the result is not true, then there is a point $x \in \partial P$ such that the orbit of $(x, \theta)$ is infinite but takes on only a finite number of angles $\left\{\theta_{i_{1}}, \ldots, \theta_{i_{n}}\right\}$. Let $I(x):=\cup e_{j} \times \theta_{i_{k}} \subset I$ be the (finite) set of intervals in the directions visited by the orbit of $(x, \theta)$.

First note that the set $I(x)$ cannot be $T$-invariant, this would contradict the fact that the $D$ orbit of $\theta$ is infinite.

Let $J^{ \pm}:=J^{ \pm}(x):=\left\{\left(x^{\prime}, \theta^{\prime}\right) \in I(x): T^{ \pm}\left(x^{\prime}, \theta^{\prime}\right) \in I(x)\right\}$. Each of these sets is a finite union of intervals and the total length of $J^{+}$is equal to the total length of $J^{-}$. Thus the total length of the intervals of $I(x)$ where the forward map is not defined is equal to the total length of the intervals where the backwards map is not defined. Thus we can (in an arbitrary manner) complete the definition of the partially defined map to an interval exchange transformation $G$ (IET). The IET $G$ agrees with the partially defined first return billiard map whenever it was defined.

Since $G$ is an IET, the well known topological decomposition holds, the interval of definition is decomposed into periodic and minimal components, with the boundary of the components consisting of saddle connections, i.e., orbits starting and ending at a point of discontinuity of the IET (see for example [MT]). Saddle connections correspond to generalized diagonals of the billiard.

Since the original billiard direction is non-exceptional the $G$-orbit of $(x, \theta)$ cannot be periodic. By the topological decomposition theorem the if orbit of $(x, \theta)$ is not all of $I(x)$ it must accumulate on a saddle connection of $G$. This saddle connection must be in the closure of the set $J^{+}$, thus it corresponds to a generalized diagonal of the billiard map. This contradicts the fact that $\theta$ is non-exceptional.

(ii) The proof is similar as for (i). Define the ghost map and its extension in the same way as above. The conclusion is just the consequence of the topological decomposition theorem for IETs.

The main result of this section follows.

Theorem 4.2. Let $\left(P ; u_{0}\right) \approx\left(Q ; v_{0}\right), P$ rational, $u_{0}$ non-exceptional. Then $Q$ is rational with $v_{0}$ non-exceptional.

Proof. All the assumptions of Lemma 3.3 are fulfilled, thus $\phi\left(C_{P}\right)=$ $C_{Q}$. By Lemma 3.7, the $v_{0}$ visits a finite number of floors.

First let us assume that $v_{0}$ is exceptional, i.e., $v_{0}$ is parallel to a generalized diagonal $d$. Let $y$, resp. $y^{\prime}$ be an outgoing, resp. incoming corner of $d$ with $y^{\prime}=\pi_{1}\left(S^{m}((y, \beta))\right)$ for some $m \in \mathbb{N}$ and a direction $\beta$ with respect to a side $f$. Since by Lemma 3.4(iv) also $v_{0}$ is recurrent and it visits a finite number of floors, there is a sequence $\{n(k)\}_{k \geq 0}$ such that 
for each $k,\left(y_{k}, \beta\right)=S^{n(k)}\left(v_{0}\right) \in \omega\left(v_{0}\right)$ with $y \neq y_{k} \in f$ and $\lim _{k} y_{k}=y$. Put $e=\phi^{-1}(f), x_{k}=\phi^{-1}\left(y_{k}\right), x=\phi^{-1}(y)$ and $x^{\prime}=\phi^{-1}\left(y^{\prime}\right)$. Then $x, x^{\prime} \in C_{P}$ and since also $u_{0}$ visits a finite number of floors, the sequence $\left\{\pi_{2}\left(\Phi^{-1}\left(\left(y_{k}, \beta\right)\right)\right)\right\}_{k \geq 0}$ has to be eventually constant, i.e., $\Phi^{-1}\left(\left(y_{k}, \beta\right)\right)=$ $\left(x_{k}, \alpha\right)=T^{n(k)}\left(u_{0}\right) \in \omega\left(u_{0}\right)$ for each sufficiently large $k$. The reader can verify that $u_{0}$ is parallel to a generalized diagonal outgoing from $x$ and incoming to $x^{\prime}$. This is impossible for $u_{0}$ non-exceptional. It shows that $v_{0}$ has to be non-exceptional.

If the polygon $Q$ were irrational, Theorem 4.1(i) would imply, since the orbit of $v_{0}$ is infinite, that $v_{0}$ is exceptional. This is impossible by Theorem 4.1.

\section{Rational VERsus Rational}

In a rational polygon a billiard trajectory may have only finitely many different directions. In Section two we introduced the invariant subset $R_{\theta}$ of the phase space consisting of the set of points whose second projection belongs to the orbit of $\theta$ under the dihedral group $D_{N} ; R_{\theta}$ has the structure of a surface. For non-exceptional $\theta$ 's the faces of $R_{\theta}$ can be glued according to the action of $D_{N}$ to obtain a flat surface depending only on the polygon $P$ but not on the choice of $\theta$ - we will denote it $R_{P}$.

Let us recall the construction of $R_{P}$. Consider $2 N$ disjoint parallel copies $P_{1}, \ldots, P_{2 N}$ of $P$ in the plane. Orient the even ones clockwise and the odd ones counterclockwise. We will glue their sides together pairwise, according to the action of the group $D_{N}$. Let $0<\theta=\theta_{1}<$ $\pi / N$ be some angle, and let $\theta_{i}$ be its $i$-th image under the action of $D_{N}$. Consider $P_{i}$ and reflect the direction $\theta_{i}$ in one of its sides. The reflected direction is $\theta_{j}$ for some $j$. Glue the chosen side of $P_{i}$ to the identical side of $P_{j}$. After these gluings are done for all the sides of all the polygons one obtains an oriented compact surface $R_{P}$.

Let $p_{i}$ be the $i$-th vertex of $P$ with the angle $\pi m_{i} / n_{i}$ and denote by $G_{i}$ the subgroup of $D_{N}$ generated by the reflections in the sides of $P$, adjacent to $p_{i}$. Then $G_{i}$ consists of $2 n_{i}$ elements. According to the construction of $R_{P}$ the number of copies of $P$ that are glued together at $p_{i}$ equals to the cardinality of the orbit of the test angle $\theta$ under the group $G_{i}$, that is, equals $2 n_{i}$.

Each corner $p \in C_{P}$ of $P$ corresponds to an angle $A(p) \in(0,2 \pi) \backslash\{\pi\}$.

Proposition 5.1. Let $\left(P ; u_{0}\right) \approx\left(Q ; v_{0}\right)$, $P$ rational with $u_{0}$ nonexceptional. Then $\phi\left(C_{P}\right)=C_{Q}$ and $A(p)=A(\phi(p))$ for each $p \in C_{P}$.

A triangle is determined (up to similarity) by its angles, thus Proposition 5.1 implies 
Corollary 5.2. Let $\left(P ; u_{0}\right) \approx\left(Q ; v_{0}\right), P$ a rational triangle with $u_{0}$ non-exceptional. Then $Q$ is similar to $P$.

Proof. Theorem 4.2 implies that also $Q$ is rational with a non-exceptional leader $v_{0}$. From Lemma 3.3 follows that $\phi\left(C_{P}\right)=C_{Q}$; let $k=\# C_{P}=$ $\# C_{Q}$, number the corners $p_{i}$ of $P$, resp. $q_{i}$ of $Q$ to satisfy $\phi\left(p_{i}\right)=q_{i}$, $i=1, \ldots, k$. Since $P, Q$ are rational and simply connected, $A\left(p_{i}\right)=$ $\pi m_{i}^{P} / n_{i}^{P}$ and $A\left(q_{i}\right)=\pi m_{i}^{Q} / n_{i}^{Q}$, where $m_{i}^{P}, n_{i}^{P}$, resp. $m_{i}^{Q}, n_{i}^{Q}$ are coprime integers. In what follows, we will show that $n_{i}^{P}=n_{i}^{Q}$ and $m_{i}^{P}=m_{i}^{Q}$.

First of all, it is known that, since $u_{0}$ is non-exceptional, the least common multiple $N_{P}$ of $n_{i}^{P}$ 's is equal to

$$
\#\left\{u \in \omega\left(u_{0}\right): \pi_{1}(u)=x\right\}=\#\left\{u \in \omega\left(u_{0}\right): \pi_{1}(u)=x^{\prime}\right\},
$$

whenever $x, x^{\prime} \in \delta P \backslash C_{P}$; moreover, if in addition $x$ and $x^{\prime}$ are from the same side then

$$
\pi_{2}\left(\left\{u \in \omega\left(u_{0}\right): \pi_{1}(u)=x\right\}\right)=\pi_{2}\left(\left\{u \in \omega\left(u_{0}\right): \pi_{1}(u)=x^{\prime}\right\}\right),
$$

The analogous equalities are true for $N_{Q}$.

Using (10) and Lemma 3.4(ii)(31) we get $N_{P}=N_{Q}=N$. Thus, both rational billiards correspond to the same dihedral group $D_{N}$.

Second, consider the local picture around the $i$ th vertex $p_{i}$. Denote the two sides which meet at $p_{i}$ by $e$ and $e^{\prime}$. Suppose there are $2 n_{i}^{P}$ copies of $P$ which are glued at $p_{i}$. Enumerate them in a cyclic counterclockwise fashion $1,2, \ldots, 2 n_{i}^{P}$. Since $u_{0}$ is non-exceptional it's orbit is minimal, so it visits each of the copies of $P$ glued at $p_{i}$. In particular the orbit crosses each of the gluings (copy $j$ glued to copy $j+1$ ).

Now consider the orbit of $v_{0}$. We need to show that there are the same number of copies of $Q$ glued at $q_{i}=\phi\left(p_{i}\right)$. Fix a $j \in\left\{1, \ldots, 2 n_{i}^{P}\right\}$ viewed as a cyclic group. Since $u_{0}$ is non-exceptional the orbit of $u_{0}$ must pass from copy $j$ to copy $j+1$ of $P$ or vice versa from copy $j+1$ to copy $j$. Suppose that we are at the instant that the orbit $u_{0}$ passes from copy $j$ to copy $j+1$ of $P$. At this same instant the orbit of $v_{0}$ passes through a side. We label the two copies of $Q$ by $j$ and $j+1$ respectively. This labeling is consistent for each crossing from $j$ to $j+1$.

Since this is true for each $j$, the combinatorial data of the orbit $u_{0}$ glue the corresponding $2 n_{i}^{P}$ copies of $Q$ together in the same cyclic manner as the corresponding copies of $P$. Note that the common point of the copies of $Q$ is a common point of $\phi(e)$ and $\phi\left(e^{\prime}\right)$ thus it is necessarily the point $q_{i}=\phi\left(p_{i}\right)$. In particular, since Lemma 3.4(iii) applies, we have $2 n_{i}^{P}$ copies of $Q$ glued around $q_{i}$ to obtain an angle which is a multiple of $2 \pi$. The total angle at this corner is by definition $2 \pi m_{i}^{Q}$. 
Thus $2 n_{i}^{Q}$ must divide $2 n_{i}^{P}$. The argument is symmetric, thus we obtain $2 n_{i}^{P}$ divides $2 n_{i}^{Q}$. We conclude that $n_{i}^{P}=n_{i}^{Q}$.

Third, let us show that $m_{i}^{Q}=m_{i}^{P}$. Realizing the gluing of $2 n_{i}^{P}$ copies of $P$ together at $p_{i}$ we get a point $p \in R_{P}$ with total angle of $2 \pi m_{i}^{P}$. If $m_{i}^{P}>1$, the point $p$ is a cone angle $2 \pi m_{i}^{P}$ singularity. In any case, for the direction $\theta$ and the corresponding constant flow on $R_{P}$, there are $m_{i}^{P}$ incoming trajectories that enter $p$ on the surface $R_{P}$, hence also $m_{i}^{P}$ points in $V_{P}$ that finish their trajectory after the first iterate at the corner $p_{i}$. Repeating all arguments for $Q$ and $\vartheta=\pi_{2}\left(v_{0}\right)$, one obtain $m_{i}^{Q}$ points in $V_{Q}$ that finish their trajectory after the first iterate at the corner $q_{i}=\phi\left(p_{i}\right)$. Since such a number has to be preserved by the homeomorphism $\Phi$, the inequality $m_{i}^{P} \neq m_{i}^{Q}$ contradicts our assumption $\left(P ; u_{0}\right) \approx\left(Q ; v_{0}\right)$. Thus, $m_{i}^{Q}=m_{i}^{P}$.

We have recalled in Section 2 that the billiard map $T$ has a natural invariant measure on its phase space given by the formula $\mu=\sin \theta \mathrm{d} x \mathrm{~d} \theta$ and the notion of a generic point $u$. In the case, when $P$ is rational and the corresponding billiard flow is dense in the surface $R_{P}$, the measure $\mu$ sits on the skeleton $K_{P}$ (union of edges) of $R_{P}$. In particular, an edge $e$ of $K_{P}$ associated with $\theta$ has the $\mu$-length $|e| \cdot \sin \theta$. As before the number $N$ is defined as the least common multiple of $n_{i}$ 's, where the the angles of a simply connected rational polygon $P$ are $\pi m_{i} / n_{i}$,

We have seen in Example 2.2 that for $N=2$ order-equivalent nonsimilar polygons exist even if they are uniquely ergodic - (note that rectangular billiards are uniquely ergodic in every non excpetional direction). For any rational polygon with $N=2$ we can speak - up to rotation - about horizontal, resp. vertical sides. Two such polygons, $P$ and $Q$ with sides $e_{i}$ resp. $f_{i}$, are affinely similar if they have the same number of corners/sides, corresponding angles equal and there are positive numbers $a, b \in \mathbb{R}$ such that $\left|e_{i}\right| /\left|f_{i}\right|=a$, resp. $\left|e_{i}\right| /\left|f_{i}\right|=b$ for any pair of corresponding horizontal, resp. vertical sides.

Theorem 5.3. Let $\left(P ; u_{0}\right) \approx\left(Q ; v_{0}\right), P$ rational, $u_{0}$ generic.

(1) If $N=N_{P} \geq 3$ then $Q$ is similar to $P$.

(2) If $N=N_{P}=2$ the $Q$ is affinely similar to $P$.

Proof. 1) We denote the sides of $P$, resp. $Q$ by $e_{i}$, resp. $f_{i}$. The generic leader $u_{0}$ is, in particular, non-exceptional. Then Theorem 4.2 claims that also $Q$ is rational with $v_{0}$ non-exceptional and by Proposition 5.1. $\phi\left(C_{P}\right)=C_{Q}$ and $A(p)=A(\phi(p))$ for each $p \in C_{P}$.

The billiard map $S$ has invariant measures defined on its phase space; one of them is given by the formula $\nu=\sin \vartheta \mathrm{d} f \mathrm{~d} \vartheta$. Consider the conjugacy $\Phi: \omega\left(u_{0}\right) \rightarrow \omega\left(v_{0}\right)$ guaranteed by Lemma 3.4(ii). Since the 
map $\Phi$ conjugates $T$ and $S$ and $\Phi\left(u_{0}\right)=v_{0}, \nu=\Phi^{*} \mu$ and $\nu$ is in fact the unique invariant measure of $S$ with $v_{0}$ generic.

Consider the reduction of the billiard map $T$ in the direction $\pi_{2}\left(u_{0}\right)$ to an interval exchange. In our case when $u_{0}$ is non-exceptional and, by Proposition 2.3, the set of directions $\Theta(e)=\pi_{2}\left(\pi^{-1}(x) \cap \omega\left(u_{0}\right)\right)$, $x \in e$, does not change on a side $e$, it consists in the union of $N$ copies of each side $e$ rescaled by the factor $\sin \theta_{i}$, where $\theta_{i} \in \Theta(e)$ is measured with respect to the direction of an oriented side $e$. Let the interval $I$ be the union of all rescaled interval-copies over all sides of $P$. The billiard map $T$ induces a piecewise isometry $T_{I}$ of the segment $I$ that can be modified [MT] to an interval exchange transformation. Using the fact that $T$ (measure $\mu$ ), resp. $S$ (measure $\nu$ ) is uniquely ergodic, the maps $T, S$ are conjugated and lengths of interval-copies are in fact $\mu$-lengths of edges on the skeleton $K_{P}$, resp. $\nu$-lengths of edges on the skeleton $K_{Q}$ and the resulting interval exchange transformations are (after an appropriate permutations of interval-copies in one of them) the same. In particular, for a side $e$ of $P$, any $\theta \in \Theta(e)$ and $(f, \vartheta)=\Phi((e, \theta))$,

$$
|e| \sin \theta=|f| \sin \vartheta \text {. }
$$

Assume that the least common multiple $N$ of the denominators of angles of $P$ is greater than or equal to 3 . Since the maps $T, S$ are conjugated, the polygons $P, Q$ correspond to the same dihedral group $D_{N}$ generated by the reflections in lines through the origin that meet at angles $\pi / N$ The orbit of $\theta_{0}^{+}=\pi_{2}\left(u_{0}\right)$, resp. $\vartheta_{0}^{+}=\pi_{2}\left(u_{0}\right)$ under $D_{N}$ consists of $2 N$ angles

$$
\theta_{j}^{+}=\theta_{0}^{+}+2 j \pi / N, \theta_{j}^{-}=\theta_{0}^{-}+2 j \pi / N,
$$

resp.

$$
\vartheta_{j}^{+}=\vartheta_{0}^{+}+2 j \pi / N, \vartheta_{j}^{-}=\vartheta_{0}^{-}+2 j \pi / N
$$

Since $N \geq 3$, for each side $e$, resp. $f$ one can find the angles

$$
\theta, \theta+2 \pi / N \in \Theta(e), \text { resp. } \vartheta, \vartheta+2 \pi / N \in \Theta(f)
$$

such that by Lemma 3.4(iii) $\Phi(e, \theta)=(f, \vartheta)$ and $\Phi(e, \theta+2 \pi / N)=$ $(f, \vartheta+2 \pi / N)$. Then as in (13) $)$

$$
|e| \sin \theta=|f| \sin \vartheta,|e| \sin (\theta+2 \pi / N)=|f| \sin (\vartheta+2 \pi / N),
$$

hence after some routine computation we get $|e|=|f|$.

2) As in part 1) the generic leader $u_{0}$ is non-exceptional, so by Theorem 4.2 also $Q$ is rational with $v_{0}$ non-exceptional. By Proposition 5.1, $\phi\left(C_{P}\right)=C_{Q}$ and $A(p)=A(\phi(p))$ for each $p \in C_{P}$. Similarly as in the mentioned proof, for a side $e$ of $P$, any $\theta \in \Theta(e)$ and 
$(f, \vartheta)=\Phi((e, \theta))=(\phi(e), \vartheta)$

$$
|e| \sin \theta=|f| \sin \vartheta
$$

where $\theta$, resp. $\vartheta$ can be taken the same for any pair of horizontal, resp. vertical sides. Thus, the number $a=|e| /|f|$, resp. $b=|e| /|f|$ does not depend on a concrete choice of a pair of $\phi$-corresponding horizonal , resp. vertical sides. This finishes the proof of our theorem.

\section{Conclusions And open questions}

Let us summarize our main results in terms of order-equivalence classes. We call an order equivalence class a non-exceptional (resp. generic) order-equivalence class if the point $u_{0}$ in $P$ is additionally supposed to be non-exceptional (resp. generic with respect to $\mu$ ). Two order equivalent polygons $P, Q$ are said to be quasisimilar if $\phi\left(C_{P}\right)=$ $C_{Q}$ and $A(p)=A(\phi(p))$ for each $p \in C_{P}$.

Theorem 6.1. Let us consider order-equivalence classes defined in Definition 2.1. Then:

- An irrational non-exceptional order-equivalence class does not contain rational polygons - Th.4.1.

- A rational non-exceptional order-equivalence class contains quasisimilar polygons - Prop 5.1.

- A rational non-exceptional triangle order-equivalence class is (up to the similarity) one point - Cor 5.2.

- A rational generic order-equivalence class with $N \geq 3$ is (up to the similarity) one point - Th5.3(1).

- A rational generic order-equivalence class with $N=2$ is (up to affine similarity) one point - Th.5.3(2).

- For each $n \geq 3,(n \neq 4)$ the order-equivalence class of the regular $n$-gon is (up to the similarity) one point.

The last part of the theorem follows by combining Theorem 5.3 with Proposition 2.4. The following questions remain open:

(1) Can one replace the assumption that both orbits are dense (Definition 2.1 (i)) by only one orbit being dense?

(2) Can one replace the assumption the orbit is generic by the orbit being non-exceptional in Theorem 5.3]

(3) Can two irrational (non-similar) polygons be order equivalent?

(4) For a rational polygon $P$, does the density of the (foot) orbit in $\partial P$ imply that the direction is non-exceptional?

Acknowledgements We gratefully acknowledge the support of the "poste tcheque" of the University of Toulon. The first author was 
also supported by MYES of the Czech Republic via contract MSM 6840770010 .

\section{REFERENCES}

[BK] K. Burns and A. Katok, Manifolds with nonpositive curvature Ergodic Theory and Dynamical Systems 5 (1985) 307-317.

[GWW] C. Gordon, D. Webb, and S. Wolpert, Isospectral plane domains and surfaces via Riemannian orbifolds, Inventiones mathematicae (1992) 1-22.

[K] M. Kac, Can one hear the shape of a drum? American Mathematical Monthly 73 (1966) 1-23.

[MT] H. Masur and S. Tabachnikov, Rational Billiards and Flat Surfaces, in Handbook of Dynamical Systems, Vol. 1A, Elsevier Science B.V., 2002, edited by B. HasselBlatt and A. Katok.

[M] J. Milnor, Eigenvalues of the Laplace operator on certain manifolds Proceedings of the National Academy of Sciences of the United States of America 51 (1964) 542.

[O] J.-P. Otal, Jean-Pierre, Le spectre marqu des longueurs des surfaces courbure ngative Ann. of Math. 131 (1990) 151-162.

[V] W. Veech, The Billiard in Regular Polygon, Geom. and Funct. Anal. (1992) 341-379.

KM FSv ČVUT, Thákurova 7, 16629 Praha 6, Czech Republic

E-mail address: bobok@mat.fsv.cvut.cz

Centre de physique théorique, Federation de Recherches des Unites de Mathematique de Marseille, Institut de mathématiques de Luminy and, Université de la Méditerranée, Luminy, Case 907, F-13288 MarSeille Cedex 9, France

E-mail address: troubetz@iml.univ-mrs.fr

URL: http://iml.univ-mrs.fr/ troubetz/ 